Vietnam Journal of Mechanics, VAST, Vol.38, No. 4 (2016), pp. 267-278

DOI:10.15625/0866-7136/6999

\title{
BODY PARAMETERS WITH APPLICATION OF OPTIMAL DIFFERENTIAL VARIATION METHOD IN SUPER CAVITY MODEL
}

\author{
Tran Thu Ha ${ }^{1,2, *}$, Nguyen Anh Son ${ }^{3}$, Duong Ngoc Hai ${ }^{1}$ \\ ${ }^{1}$ Institute of Mechanics, Vietnam Academy of Science and Technology, Hanoi, Vietnam \\ ${ }^{2}$ VNU University of Engineering and Technology, Hanoi, Vietnam \\ ${ }^{3}$ National University of Civil Engineering, Hanoi, Vietnam \\ *E-mail: tran_thuha1@yahoo.com \\ Received September 16, 2015
}

\begin{abstract}
When a slender body moves very fast through water at sufficient speed the cavity phenomena is happened. In a cavity model the bodys lengths and cavitator diameter are determined by the differential variation optimal method so that the velocity of body is maximum.
\end{abstract}

Keywords: Super cavity, Runge-Kutta methods, data assimilation.

\section{INTRODUCTION}

In hydrodynamics applications cavitation is the appearance of vapor bubbles and pockets inside homogeneous liquid medium. This phenomenon occurs because the pressure is reduced to the vapor pressure limit. We will study super cavity appearing by the very fast movement of slender body in water that makes uncontrolled gun-launched slender body. Except the body head called by cavitator is directly touching with water, the gas layer can be covered partial or full body depending on the design of body form. The body rotates about its nose. In this paper for simple calculation we choose cavitator formed by the plate disk with diameter (see Fig. 1). The body is consisted of two parts: the cone top and cylinder part with the diameter $d$.

In the super cavity model the following assumptions are (see [1]):

- The motion of the projectile is confined to a plane;

- The slender body rotates about its nose;

- The effect of gravity on the dynamics of this body is negligible;

- The motion of the slender body is not influenced by the presence of gas, water vapor or water drops in the cavity;

(C) 2016 Vietnam Academy of Science and Technology 


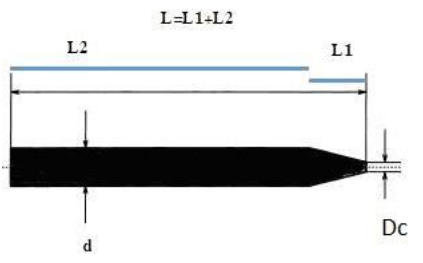

- $L$ is the length of the slender body

- $L_{2}$ is the body's length of cylinder part

- $L_{1}$ is the body's length of cone top part

- $d$ is the body's diameter

- $D_{c}$ is the body's cavitator diameter

Fig. 1. Slender body geometery

The super cavity problems are studied in ( [1-8]).

The Data Assimilation method using differential variation is based on the theory of optimal control for partial differential equation (Lions, 1971 see [9-11]). This method is applied to correct and find coefficients, solve the inverse problems, simulate the air and fluid pollution processes (see [12-18]). In this paper by applying the differential variation optimal method we will concentrate the study on the identification parameters $L_{1}, L_{2}, D_{c}$ so that the distance way running by body is maximum.

\section{GENERAL VARIATIONAL APPROACH}

By the differential variation optimal method the length and cavitator diameter parameters $L_{1}, L_{2}, D_{c}$ will be evaluated as the solution of an "Inverse Problem", basically as the solution of an optimization problem. The advantage is that there exist many efficient algorithms for solving this problem. Most of them require to compute the gradient of the function to be minimized. The cost function is done by solving an "Adjoin Model". The method is described in many papers together with the computational developments (see [9-18]). It can be summarized as follows:

Consider the mathematical equation of physic process that is described by the evolution problem: Let $X(t)$ the state vector describing the evolution of a system governed by the abstract equation

$$
\frac{d X}{d t}=F\left(X, E_{1}, \ldots, E_{n}\right), \quad X(0)=X_{0},
$$

where $E_{1}, \ldots, E_{n}$ are the equation parameters; $X(t)$ is a unknown state vector belonging for any $t$ to a Hilbert space $\Im, X_{0} \in \Im$; $F$ is a nonlinear operator mapping $Y \times Y_{p}$ to $Y$ with $Y=L_{2}(0, T, \Im),\|\cdot\|_{Y}=(., .)_{Y}^{1 / 2}, Y_{p}$ is Hilbert space (the space of model's parameters); Suppose that for given initial value $X(0)=X_{0} \in \Im$ and $\left(E_{1}, \ldots, E_{n}\right) \in Y_{p}$ there exists a unique solution $X \in \Im$ to (1). To choose $E_{1}, \ldots, E_{n}$ so that the function $\int_{0}^{T} H(X) d t$ is getting maximum in running process $(0, T)$ we introduce the functional called cost function

$$
J\left(E_{1}, \ldots, E_{n}\right)=-\int_{0}^{T} H(X) d t+\frac{1}{2} \sum_{i=1}^{n}\left\|E_{i}-E_{i, 0}\right\|^{2} .
$$


Here $E_{1,0}, \ldots, E_{n, 0}$ are priori evaluations of $E_{1}, \ldots, E_{n}$. The problem is to determine $E_{1}^{*}, \ldots, E_{n}^{*}$ by minimizing $J$. The second and the third terms in $J$ are a regularization term, in the sense of Tykhonov, have a well posed problem (see $[14,18])$.

The optimal solutions are characterized by $\vec{\nabla} . J\left(E_{1}^{*}, \ldots, E_{n}^{*}\right)$, where $\vec{\nabla}$.J is the gradient of $J$. To compute this gradient we introduce $\bar{E}_{i}(i=1, \ldots, n)$ the directions in the space $Y_{p}$. We will compute the Gateaux derivative of the cost function $J$ by $E_{i}$ in the directions of $\bar{E}_{i}$.

The Gateaux derivative of the cost function $J$ in the directions of $\bar{E}_{i}(i=1, \ldots, n)$ will be

$$
\begin{aligned}
\hat{J}\left(E_{1}, \ldots, E_{n}\right) & =-\sum_{i=1}^{n} \int_{0}^{T}\left(\frac{\partial H}{\partial X}, \hat{X}^{(i)}\right)_{\Im} d t+\sum_{i=1}^{n}\left\langle E_{i}-E_{i, 0}, \bar{E}_{i}\right\rangle \\
& =-\sum_{i=1}^{n} \int_{0}^{T}\left(G(X), \hat{X}^{(i)}\right)_{\Im} d t+\sum_{i=1}^{n}\left\langle E_{i}-E_{i, 0}, \bar{E}_{i}\right\rangle,
\end{aligned}
$$

where $G(X)=\frac{\partial H}{\partial X}$ and $\hat{X}^{(i)}, \hat{J}_{E_{i}}\left(E_{1}, \ldots, E_{n}\right)$ respectively are the Gateaux derivatives of $X$ and $J$ with respect to $E_{i}$ in the directions $\bar{E}_{i}$. Here $<_{,}>$is the dot product associated with the norm operator \|\| . The optimal solution of problem is characterized by $\hat{J}\left(E_{1}, \ldots, E_{n}\right)=\vec{\nabla} . J .\left(\bar{E}_{1}, \ldots, \bar{E}_{n}\right)^{T}=0$ where $\vec{\nabla} \cdot J=\left(J_{E_{1}}^{\prime}, \ldots, J_{E_{n}}^{\prime}\right)$ is the gradient of $J$ with respect to $E_{1}, \ldots, E_{n}$.

The superscript ${ }^{T}$ indicates the transpose of the vector. To find $J_{E_{i}}^{\prime}\left(E_{1}, \ldots, E_{n}\right)$ the same way as in ( $[9-13,15-18])$ we solve the adjoin equation of (1)

$$
\frac{d P^{(i)}}{d t}+\left[\frac{d F}{d X}\right]^{t} \cdot P^{(i)}=G(X), \quad P^{(i)}(T)=0 .
$$

Then by the similar way as in $([9-13,15-18])$ we have

$$
J_{E_{i}}^{\prime}\left(E_{1}, \ldots, E_{n}\right)=\int_{0}^{T}\left[\frac{\partial F}{\partial E_{i}}\right]^{t} P^{(i)} d t+E_{i}-E_{i, 0}
$$

Eqs. (1)-(5) are the Optimality System (O.S). The adjoin model will be run back word to get the gradient which are used to carry out an algorithm of optimization (see $[9-13,15-18])$.

\section{MATHEMATICAL MODEL FOR THE BODY MOTION}

To describe the motion of body, a body fixed coordinate system as shown in Fig. 2 is chosen. $\left(X_{0}, Y_{0}, Z_{0}\right)$ is the inertial reference frame with origin at 0 and $\left(X_{1}, Y_{1}, Z_{1}\right)$ is the non-inertial reference frame with origin at $A$, the tip of the slender body. The $X_{1}$ axis coincides with the longitudinal axis of the slender body. The components of velocity of point $A$ along $X_{0}$ and $Z_{0}$ direction are $U_{F}$ and $W_{F}$ respectively. The components of velocity of point $A$ along $X_{1}$ and $Z_{1}$ direction are $U$ and $W$ respectively. The angular 
velocity about $Y_{0}$ axis is $Q$. The orientation angle of the body with respect to the $Y_{0}$ axis is $\vartheta$.

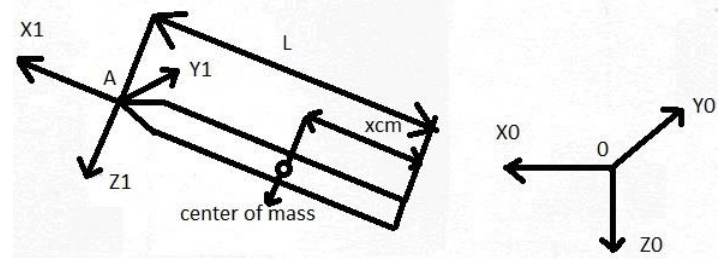

Fig. 2. Axes of body and inertial frames

The relationships between body and inertial fixed velocities are described by the following formula (see [19])

$$
U_{F}=U \cos \vartheta+W \sin \theta, \quad W_{F}=-U \sin \vartheta+W \cos \vartheta, \quad \dot{\vartheta}=Q, \quad \vartheta(0)=\vartheta_{0} .
$$

The mathematic cavity model (see [20]) is used to describe the motion of slender body under water in cavity. The motion of slender body in both phases is written by the following equations:

Phase 1: For $U^{2} \gg W^{2}$ and $\rho A_{c} k(U, W, h) U^{2} \gg 2 m L Q^{2}$ the equation can be written as

$$
\begin{aligned}
& \frac{\partial U}{\partial t}=-\frac{1}{2 m} \rho k(U, W, h) A_{c} U^{2}, \quad \frac{\partial W}{\partial t}=Q U, \quad \frac{\partial Q}{\partial t}=0, \\
& \frac{\partial h}{\partial t}=-U \sin \vartheta+W \cos \vartheta, \quad \frac{\partial \vartheta}{\partial t}=Q, \\
& U(0)=U_{0}, \quad W(0)=W_{0}, \quad Q(0)=Q_{0}, \quad h(0)=h_{0}, \quad \vartheta(0)=\vartheta_{0} .
\end{aligned}
$$

Phase 2: For $U^{2} \gg W^{2}$ and $\rho A_{c} k(U, W, h) U^{2} \gg 2 m L Q^{2}$ the equation can be written as

$$
\begin{aligned}
& \frac{\partial U}{\partial t}=-\frac{1}{2 m} \rho k(U, W, h) F\left(A_{c}, r, l_{k}, \theta\right) U^{2}, \\
& \frac{\partial W}{\partial t}=K W^{2}\left[M_{1} l_{k}+M_{2} l_{k} x_{c m}\left(L-x_{c m}\right)\right]+2 K W\left[Q M_{2} L x_{c m} l_{k}\left(L-x_{c m}\right)\right]+Q U, \\
& \frac{\partial Q}{\partial t}=-K M_{2}\left[W^{2} l_{k} x_{c m}+2 W Q L l_{k} x_{c m}\right], \quad \frac{\partial h}{\partial t}=-U \sin \vartheta+W \cos \vartheta, \quad \frac{\partial \vartheta}{\partial t}=Q,
\end{aligned}
$$

where $\theta$ is the angle of slender body during impact with the cavity boundary,

$$
\begin{aligned}
& \tan \theta \approx \frac{W}{U} \text { or } \theta \approx \arctan \frac{W}{U}, M_{1}=-\frac{\rho d}{m}, M_{2}=\frac{\rho d}{I}, \\
& F\left(A_{c}, r, l_{k}, \theta\right)=A_{c}+r^{2} \cos ^{-1}\left(\frac{r-l_{k} \tan \theta}{r}\right)-\left(r-l_{k} \tan \theta\right) \sqrt{d l_{k} \tan \theta} \\
& k(U, W, h)=k_{1} C_{D 0}(1+\sigma) \cos ^{2} \alpha, C_{D 0}=0.82,
\end{aligned}
$$

$\alpha$ is the angle between flow direction and body's direction in moving, $\cos \alpha \approx$ $U^{\prime} \sqrt{U^{2}+W^{2}}, p_{\infty}=\rho g h+P_{a t m}$ ambient pressure, $l_{k}$ is the wetted length of the body; $k_{1}, K$ are parameters, $h$ is the water depth between the body's position and water free surface, $\rho$ is the mass density of water, $x_{c m}$ is the distance between body's tail and it's center of mass, 
$m$ is the mass of the slender body, $\sigma$ is the cavitation number $\sigma=2\left(p_{\infty}-p_{c}\right) /\left(U^{2}+W^{2}\right)$, $I$ is the moment of inertia of the body about an axis parallel to the $Y_{1}$ axis and passing through its center of mass, $r=d / 2$ is the radius of slender body, $A_{c}=\pi D_{c}^{2} / 4$ is the area of the cavitator, $r_{c}=D_{c} / 2$ is the cavitator radius, $g=9.81 \mathrm{~m} / \mathrm{s}$ is the gravity acceleration, $p_{c}$ is the vapor pressure of water.

To get the above equations the following condition is needed: $l_{k} / L \ll 1$. The geometry of the cavity is given by (see $[1,6,8]): 4(x-l / 2)^{2} / l^{2}+4 y^{2} /\left(D_{k} / 2\right)^{2}=1$, where the maximum diameter $D_{k}$ and length $l$ of the cavity shape are given by the following formulas: $D_{k}=D_{c} \sqrt{k_{1} C_{D 0}(1+\sigma) / \sigma}, l=D_{c} \sqrt{\log (1 / \sigma)} / \sigma$.

The depending on $L$ and $D_{c}$ of $I_{y}, m$ and $x_{c m}$ can be calculated by the following formulas:

$$
\begin{aligned}
& m=\rho_{\text {body }} V, \quad x_{c m}=L_{2}+\pi\left(0.5 \cdot R^{2}\left(L_{1}^{2}-L_{2}^{2}\right)-L_{1}^{2}\left(R-0.5 \cdot D_{c}\right)\left(5 R+1.5 D_{c}\right) / 12\right) / V, \\
& I_{y}=\rho \pi\left[\begin{array}{l}
\left(L_{1}^{3} R^{2} / 3+0.2 . L_{1}^{3}\left(R-0.5 \cdot D_{c}\right)^{2}-0.5 . L_{1}^{3} R\left(R-0.5 \cdot D_{c}\right)\right)+\left(R^{2} L_{2}^{3} / 3+R^{4} L_{2} / 4\right) \\
-V x_{G}^{2} / \pi+0.25\left(R^{4} L_{1}+L_{1}\left(R-0.5 . D_{c}\right)^{4} / 5+2 R^{2} L_{1}\left(R-0.5 . D_{c}\right)^{2}\right. \\
\left.-2 R^{3} L_{1}\left(R-0.5 \cdot D_{c}\right)-R L_{1}\left(R-0.5 . D_{c}\right)^{3}\right)
\end{array}\right], \\
& V=\pi\left[R^{2}\left(L_{1}+L_{2}\right)+\left[L_{1}\left(R-0.5 D_{c}\right)^{2} / 3-R L_{1}\left(R-0.5 D_{c}\right)\right],\right. \\
& x_{G}=\pi\left(R^{2}\left(l_{1}^{2}-l_{2}^{2}\right) / 2-l_{1}^{2}\left(R-0.5 \cdot D_{c}\right)\left(5 R+1.5 D_{c}\right) / 12\right) / V,
\end{aligned}
$$

Eqs. (7)-(8) can be rewritten as follows

$$
\partial X / \partial t=A(X), \quad X(0)=X_{0},
$$

where $X=(U, W, Q, h, \vartheta)^{T}$ is an unknown state function vector of Eqs. (7)-(8), and

$$
\begin{aligned}
& X_{0}=\left(U_{0}, W_{0}, Q_{0}, h_{0}, \vartheta_{0}\right)^{T}, \\
& A(X)=\left[A_{1}(X), A_{2}(X), A_{3}(X),-U \sin \vartheta+W \cos \vartheta, Q\right]^{T}, \\
& A_{1}(X)= \begin{cases}-0.5 \rho k(U, W, h) A_{c} U^{2} / m, & \text { in the firstphase } \\
-0.5 \rho k(U, W, h) F\left(A_{c}, r, l_{k}, \theta\right) U^{2} / m, & \text { in the second phase }\end{cases} \\
& A_{2}(X)= \begin{cases}Q U, & \text { in the first phase } \\
K C_{1} W^{2}+K C_{2} W+Q U, & \text { in the second phase }\end{cases} \\
& A_{3}(X)= \begin{cases}Q U, & \text { in the first phase } \\
C_{3} W^{2}+C_{4} W Q, & \text { in the second phase }\end{cases} \\
& C_{1}=M_{1} l_{k}+M_{2} l_{k} x_{c m}\left(L-x_{c m}\right), \quad C_{2}=2 M_{2} L x_{c m} l_{k}\left(L-x_{c m}\right), \\
& C_{3}=-M_{2} l_{k} x_{c m}, \quad C_{4}=-M_{2} L l_{k} x_{c m},
\end{aligned}
$$

\section{DETERMINATION OF OPTIMAL GEOMETRIC PARAMETERS OF BODY}

By the shape of slender body the relations of $L_{1}, L_{2}, D_{c}, m, x_{c m}$ and $I$ are built by the functions such as: $m=m\left(L_{1}, L_{2}, D_{c}\right), x_{c m}=x_{c m}\left(L_{1}, L_{2}, D_{c}\right)$ and $I=I\left(L_{1}, L_{2}, D_{c}\right)$.

We will find $L_{1}^{*}, L_{2}^{*}, D_{c}^{*}$ so that the way distance by $X_{0}$ direction running by the body described by the function $\int_{0}^{T} U_{F}(t) d t$ is maximum. Firstly, we have approximations 
$L_{1,0}, L_{2,0}$ and $D_{c, 0}$ of $L_{1}, L_{2}$ and $D_{c}$. Using the formula (1) we introduce the cost function

$$
\begin{aligned}
& J\left(L_{1}, L_{2}, D_{c}\right)=-\int_{0}^{T} U_{F} d t+\left(L_{1}-L_{1,0}\right)^{2} / 2+\left(L_{2}-L_{2,0}\right)^{2} / 2+\left(D_{c}-D_{c, 0}\right)^{2} / 2 \\
& =-\int_{0}^{T}(U \cos \vartheta+W \sin \vartheta) d t+\left(L_{1}-L_{1,0}\right)^{2} / 2+\left(L_{2}-L_{2,0}\right)^{2} / 2+\left(D_{c}-D_{c, 0}\right)^{2} / 2 .
\end{aligned}
$$

Using the cost function from the formula (11) the continuous problem is to determine $L_{1}^{*}, L_{2}^{*}, D_{c}^{*}$ minimizing $J\left(L_{1}, L_{2}, D_{c}\right)$. The optimal problem is written in the form

$$
\left\{\begin{array}{l}
\partial X / \partial t=A(X), \quad X(0)=X_{0} \\
J\left(L_{1}^{*}, L_{2}^{*}, D_{c}^{*}\right)=\inf _{L_{1}^{*}, L_{2}^{*}, D_{c}^{*}} J\left(L_{1}, L_{2}, D_{c}\right)
\end{array}\right.
$$

4.1. Computing the gradient $\vec{\nabla} . J\left(L_{1}, L_{2}, D_{c}\right)$ of the cost function $J$ by $L_{1}, L_{2}$ and $D_{c}$ parameters in the directions of $\bar{L}_{1}, \bar{L}_{2}, \bar{D}_{c}$

Let $\bar{L}_{1}, \bar{L}_{2}$ and $\bar{D}_{c}$ be some functions in the space of the control. The Gateaux derivative of the cost function $J$ with respect to $L$ and $D_{c}$ in the directions of $\bar{L}$ and $\bar{D}_{c}$ will be

$$
\begin{aligned}
\hat{J}\left(L_{1}, L_{2}, D_{c}\right)= & -\int_{0}^{T}\left(G(X), \hat{X}_{1}\right)_{\Im} d t+\left(L_{1}-L_{1,0}\right) \cdot \bar{L}_{1}-\int_{0}^{T}\left(G(X), \hat{X}_{2}\right)_{\Im} d t+\left(L_{2}-L_{2,0}\right) \cdot \bar{L}_{2} \\
& -\int_{0}^{T}\left(G(X), \hat{X}_{3}\right)_{\Im} d t+\left(D_{c}-D_{c, 0}\right) \bar{D}_{c}
\end{aligned}
$$

with

$$
G(X)=\left[\begin{array}{lllll}
\cos \vartheta & \sin \vartheta & 0 & 0 & -U \sin \vartheta+W \cos \vartheta
\end{array}\right]^{T}
$$

and $\hat{X}^{(i)}=\left(\hat{U}^{(i)}, \hat{W}^{(i)}, \hat{Q}^{(i)}, \hat{h}^{(i)}, \hat{\vartheta}^{(i)}\right)^{T}(i=1,3)$ respectively are the Gateaux derivatives of $X=(U, W, Q, h, \vartheta)^{T}$ by $L_{1}, L_{2}$ and $D_{c}$ in the directions of $\bar{L}_{1}, \bar{L}_{2}, \bar{D}_{c}$.

Firstly, we will compute Gateaux derivatives $\hat{J}_{L_{1}}\left(L_{1}, L_{2}, D_{c}\right), \hat{J}_{L_{2}}\left(L_{1}, L_{2}, D_{c}\right)$ and $\hat{J}_{D_{c}}\left(L_{1}, L_{2}, D_{c}\right)$ of the cost function $J$ with respect to $L_{1}, L_{2}$ and $D_{c}$ respectively in the directions of $\bar{L}_{1}, \bar{L}_{2}$ and $\bar{D}_{c}$

The Gateaux derivative equations of (9) with respect to body length parameter $L_{i}(i=1,2)$ in the directions of $\bar{L}_{i}$ are written as follows

$$
\partial \hat{X}^{(i)} / \partial t=N(X) \hat{X}^{(i)}+B(X) \bar{L}_{i}, \quad \hat{X}^{(i)}(0)=0,
$$


where

$$
\begin{aligned}
& N(X)=\left[\begin{array}{ccccc}
N_{11}(X) & N_{12}(X) & 0 & N_{14}(X) & 0 \\
N_{21}(X) & N_{22}(X) & N_{23}(X) & 0 & 0 \\
0 & N_{32}(X) & N_{33}(X) & 0 & 0 \\
-\sin \vartheta & \cos \vartheta & 0 & 0 & -U \cos \vartheta-W \sin \vartheta \\
0 & 0 & 1 & 0 & 0
\end{array}\right] \\
& N_{i j}=\left\{\begin{array}{ll}
N_{i j}^{(1)}, & \text { in the first phase } \\
N_{i j}^{(2)}, & \text { in the second phase }
\end{array}(i=1, \ldots, 3 ; j=1, \ldots, 4)\right. \\
& N_{11}^{(1)}=-\frac{1}{2 m} \rho k_{1} C_{D 0}\left(1+\frac{p_{\infty}-p_{c}}{0.5 \rho\left(U^{2}+W^{2}\right)}\right) \frac{\left(2 U^{4}+3 U^{2} W^{2}\right)}{\left(U^{2}+W^{2}\right)^{3 / 2}} A_{c}+\frac{1}{m} k_{1} \rho C_{D 0} \frac{p_{\infty}-p_{c}}{0.5 \rho\left(U^{2}+W^{2}\right)^{5 / 2}} U^{4} A_{c} \text { ， } \\
& N_{12}^{(1)}=-\frac{1}{2 m} \rho k_{1} C_{D 0}\left(1+\frac{p_{\infty}-p_{c}}{0.5 \rho\left(U^{2}+W^{2}\right)}\right) \frac{U^{3} W}{\left(U^{2}+W^{2}\right)^{3 / 2}} A_{c}+\frac{1}{m} k_{1} \rho C_{D 0} \frac{p_{\infty}-p_{c}}{0.5 \rho\left(U^{2}+W^{2}\right)^{5 / 2}} W U^{3} A_{c} \text { ， } \\
& N_{14}^{(1)}=-\frac{\rho}{2 m} k_{1} C_{D 0} \frac{g}{0.5\left(U^{2}+W^{2}\right)^{3 / 2}} U^{3} A_{c} N_{21}^{(1)}=Q, N_{22}^{(1)}=0, N_{23}^{(1)}=U, N_{32}^{(1)}=0, N_{33}^{(1)}=0 \text { ， } \\
& N_{11}^{(2)}=-\frac{1}{2 m} k_{1} \rho C_{D 0}\left(1+\frac{p_{\infty}-p_{c}}{0.5 \rho\left(U^{2}+W^{2}\right)}\right) \frac{\left[\left(2 U^{4}+3 U^{2} W^{2}\right)\right] F_{c}}{\left(U^{2}+W^{2}\right)^{3 / 2}}+\frac{1}{m} k_{1} \rho C_{D 0} \frac{p_{\infty}-p_{c}}{0.5 \rho\left(U^{2}+W^{2}\right)^{5 / 2}} U^{4} F_{c} \\
& +\frac{\rho}{2 m} k_{1} C_{D 0}\left(1+\frac{p_{\infty}-p_{c}}{0.5 \rho\left(U^{2}+W^{2}\right)}\right)\left(r^{2} \frac{\sin \left(\frac{r-l_{k} \tan \theta}{r}\right) \frac{l_{k}}{r}}{\cos ^{2}\left(\frac{r-l_{k} \tan \theta}{r}\right)}-\frac{r \sqrt{\frac{l_{k} d}{\tan \theta}}}{2}+\frac{3}{2} l_{k} \sqrt{d l_{k} \tan \theta}\right) \frac{U W}{\sqrt{\left(U^{2}+W^{2}\right)}}, \\
& N_{12}^{(2)}=-\frac{1}{2 m} k_{1} \rho C_{D 0}\left(1+\frac{p_{\infty}-p_{c}}{0.5 \rho\left(U^{2}+W^{2}\right)}\right) \frac{\left[-U^{3} W \hat{W}\right] F_{c}}{\left(U^{2}+W^{2}\right)^{3 / 2}}+\frac{1}{m} k_{1} \rho C_{D 0} \frac{p_{\infty}-p_{c}}{0.5 \rho\left(U^{2}+W^{2}\right)^{5 / 2}} W U^{3} F_{c} \\
& -\frac{\rho}{2 m} k_{1} C_{D 0}\left(1+\frac{p_{\infty}-p_{c}}{0.5 \rho\left(U^{2}+W^{2}\right)}\right)\left(r^{2} \frac{\sin \left(\frac{r-l_{k} \tan \theta}{r}\right) \frac{l_{k}}{r}}{\cos ^{2}\left(\frac{r-l_{k} \tan \theta}{r}\right)}-\frac{r \sqrt{\frac{l_{k} d}{\tan \theta}}}{2}+\frac{3}{2} l_{k} \sqrt{d l_{k} \tan \theta}\right) \frac{U^{2}}{\sqrt{\left(U^{2}+W^{2}\right)}}, \\
& N_{14}^{(2)}=-\frac{\rho}{2 m} k_{1} C_{D 0} \frac{g}{0.5\left(U^{2}+W^{2}\right)^{3 / 2}} U^{3} F_{C}, \quad N_{33}^{(2)}=K C_{4} W ， \\
& N_{21}^{(2)}=Q, \quad N_{22}^{(2)}=2 K C_{1} W+K C_{2} Q, \quad N_{23}^{(2)}=K C_{2} W+U N_{32}^{(2)}=2 K C_{3} W+K C_{4} Q, \\
& B^{(i)}=\left(B_{1}^{(i)}, B_{2}^{(i)}, B_{3}^{(i)}, 0,0\right)^{T}, \\
& B_{1}^{i}= \begin{cases}\frac{1}{2 m^{2}} k(U, W, h) U^{2} A_{c} m_{L_{1}}^{\prime}, & \text { for the first phase } \\
\frac{1}{2 m} k(U, W, h) U^{2}\left(\frac{F_{c} m_{L_{1}}^{\prime}}{m}-F_{c, l_{k}}^{\prime} l_{k, L_{1}}^{\prime}\right), & \text { for the second phase }\end{cases} \\
& F_{c, l_{k}}^{\prime}=\left(r^{2} \frac{\sin \left(\frac{r-l_{k} \tan \theta}{r}\right) \frac{\tan \theta}{r}}{\cos ^{2}\left(\frac{r-l_{k} \tan \theta}{r}\right)}-\frac{r \sqrt{\frac{d \tan \theta}{l_{k}}}}{2}+\frac{3}{2} \tan \theta \sqrt{d l_{k} \tan \theta}\right),
\end{aligned}
$$




$$
\begin{aligned}
& B_{2}^{(i)}= \begin{cases}0, & \text { for the first phase } \\
C_{1, L_{i}}^{\prime} W^{2}+C_{2, L_{i}}^{\prime} W Q, & \text { for the second phase }\end{cases} \\
& B_{3}^{(i)}= \begin{cases}0, & \text { for the first phase } \\
C_{3, L_{i}}^{\prime} W^{2}+C_{4, L_{i}}^{\prime} W Q, & \text { for the second phase }\end{cases}
\end{aligned}
$$

$C_{1, L_{i}}^{\prime} C_{2, L_{i}}^{\prime} C_{3, L_{i}}^{\prime} C_{4, L_{i}}^{\prime}$ are the derivatives of those functions by length parameter $L_{i}$. Multiplying Eq. (15) by adjoin variable $P^{(i)}=\left(P_{1}^{(i)}, P_{2}^{(i)}, P_{3}^{(i)}, P_{4}^{(i)}, P_{5}^{(i)}\right)^{T}(i=1,2)$ in the same space as $X$ and then integrating by $t$ between 0 and $T$ and adding them we have

$$
\begin{aligned}
& \sum_{i=1}^{2}\left(\hat{X}^{(i)}(T), P^{(i)}(T)\right)_{\Im}-\left(\hat{X}^{(i)}(0), P^{(i)}(0)\right)_{\Im}= \\
& \sum_{i=1}^{2} \int_{0}^{T}\left(\hat{X}^{(i)}, \frac{d P^{(i)}}{d t}+F^{(i)}\left(X, P^{(i)}\right)\right)_{\Im} d t-\sum_{i=1}^{2} \bar{L}_{i} \int_{0}^{T} B^{(i)} \cdot P^{(i)^{T}} d t
\end{aligned}
$$

where $F^{(i)}\left(X, P^{(i)}\right)=N^{t} . P^{(i)}$ with $N(X)$ is defined by the formula (16).

By the similar way the Gateaux derivative equations of (7)-(8) with respect to cavitator diameter parameter $D_{c}$ in the directions of $\bar{D}_{c}$ are written as follows

$$
\partial \hat{X}^{(3)} / d t=N(X) \hat{X}^{(3)}+B^{(3)}(X) \bar{D}_{c}, \quad \hat{X}^{(3)}(0)=0,
$$

where

$$
\begin{aligned}
& B^{(3)}=\left(B_{1}^{(3)}, B_{2}^{(3)}, B_{3}^{(3)}, 0,0\right)^{T}, \\
& B_{1}^{(3)}= \begin{cases}0.5 \cdot k(U, W, h) U^{2} A_{c} m^{\prime} D_{c} / m^{2}, & \text { for the first phase } \\
0.5 \cdot k(U, W, h) U^{2}\left(F_{c} m^{\prime} D_{c} / m-F_{c, l_{k}}^{\prime} l_{k, D_{c}}^{\prime}\right) / m, & \text { for the second phase }\end{cases} \\
& B_{2}^{(3)}= \begin{cases}0, & \text { for the first phase } \\
C_{1, D_{c}}^{\prime} W^{2}+C_{2, D_{c}}^{\prime} W Q, & \text { for the second phase }\end{cases} \\
& B_{3}^{(3)}= \begin{cases}0, & \text { for the first phase } \\
C_{3, D_{c}}^{\prime} W^{2}+C_{4, D_{c}}^{\prime} W Q, & \text { for the second phase }\end{cases}
\end{aligned}
$$

Here $C_{1, D_{c}}^{\prime}, C_{2, D_{c^{\prime}}}^{\prime} C_{3, D_{c^{\prime}}}^{\prime} C_{4, D_{c}}^{\prime}, m_{D_{c}}^{\prime}$ are the derivatives of the functions $C_{1}, C_{2}, C_{3}, C_{4}, m$ by the parameter $D_{c}$. Multiplying Eq. (18) by adjoin variable $P^{(3)}=\left(P_{1}^{(3)}, P_{2}^{(3)}, P_{3}^{(3)}, P_{4}^{(3)}, P_{5}^{(3)}\right)^{T}$ in the same space as $X$ and then integrating by t between 0 and $T$ we have

$$
\begin{aligned}
& \left(\hat{X}^{(3)}(T), P^{(3)}(T)\right)_{\Im}-\left(\hat{X}^{(3)}(0), P^{(3)}(0)\right)_{\Im}= \\
& \quad \int_{0}^{T}\left(\hat{X}^{(3)}, \frac{d P^{(3)}}{d t}+F^{(3)}\left(X, P^{(3)}\right)\right)_{\Im} d t-\bar{D}_{c} \int_{0}^{T} P^{(3)^{T}} B^{(3)} d t,
\end{aligned}
$$


where $F^{(3)}\left(X, P^{(3)}\right)=N^{t} \cdot P^{(3)}$. Adding (17) and (19) we have

$$
\begin{aligned}
& \sum_{i=1}^{3}\left(\hat{X}^{(i)}(T), P^{(i)}(T)\right)_{\Im}-\left(\hat{X}^{(i)}(0), P^{(i)}(0)\right)_{\Im}= \\
& \quad \sum_{i=1}^{3} \int_{0}^{T}\left(\hat{X}^{(i)}, d P^{(i)} / d t+F^{(i)}\left(X, P^{(i)}\right)\right)_{\Im} d t-\sum_{i=1}^{2} \bar{L}_{i} \int_{0}^{T} B^{(i)} \cdot P^{(i)^{T}} d t-\bar{D}_{c} \int_{0}^{T} B^{(3)} \cdot P^{(3)^{T}} d t .
\end{aligned}
$$

Then if $P^{(i)}(i=1,3)$ are the solutions of the following equations

$$
d P^{(i)} / d t+F^{(i)}\left(X, P^{(i)}\right)=G(X), \quad P^{(i)}(T)=0 .
$$

The Eq. (20) becomes

$$
\sum_{i=1}^{3} \int_{0}^{T}\left(\hat{X}^{(i)}, G(X)\right)_{\Im} d t=\sum_{i=1}^{2} \bar{L}_{i} \int_{0}^{T} B^{(i)} \cdot P^{(i) T} d t+\bar{D}_{c} \int_{0}^{T} B^{(3)} \cdot P^{(3)^{T}} d t .
$$

Applying the Eq. (22) to the Eq. (13) we have

$$
\hat{J}\left(L_{1}, L_{2}, D_{c}\right)=\vec{\nabla} \cdot J \cdot\left(\bar{L}_{1}, \bar{L}_{2}, \bar{D}_{c}\right)^{T},
$$

where

$$
\begin{aligned}
& \vec{\nabla} . J\left(L_{1}, L_{2}, D_{c}\right)=\left(J_{L_{1}}^{\prime}\left(L_{1}, L_{2}, D_{c}\right), J_{L_{2}}^{\prime}\left(L_{1}, L_{2}, D_{c}\right), J_{D_{c}}^{\prime}\left(L_{1}, L_{2}, D_{c}\right)\right), \\
& J_{L_{i}}^{\prime}\left(L_{1}, L_{2}, D_{c}\right)=-\bar{L}_{i} \int_{0}^{T} B^{(i)} \cdot P^{(i) T} d t+\left(L_{i}-L_{i, 0}\right) \cdot \bar{L}_{i}, \quad(i=1,2), \\
& J_{D_{c}}^{\prime}\left(L_{1}, L_{2}, D_{c}\right)=-\bar{D}_{c} \int_{0}^{T} B^{(3)} \cdot P^{(3)^{T}} d t+\left(D_{c}-D_{c, 0}\right) \bar{D}_{c} .
\end{aligned}
$$

\subsection{Algorithm to solve the optimal problem}

- Solve Eqs. (9) by Runge-Kutta methods

- Solve the adjoint Eq. (21)

- Get the function $J_{L_{i}}^{\prime}(i=1,2)$ by the formula (24)

- Get the function $J_{D_{c}}^{\prime}$ by the formula (25)

- Calculate the gradient $\vec{\nabla} . J\left(L_{1}, L_{2}, D_{c}\right)$ of the cost function $J\left(L_{1}, L_{2}, D_{c}\right)$ by the formula (23)

- Solve the optimal problem (12) with the gradient $\vec{\nabla} . J\left(L_{1}, L_{2}, D_{c}\right)$

\subsection{Simulation experiment on finding the parameters $L_{1}, L_{2}$ and $D_{c}$ so that velocity $U$ is maximum}

We will consider the body with $d=0.57 \mathrm{~cm}, \rho_{\text {body }}=7680 \mathrm{~kg} / \mathrm{m}^{3}, L_{1} \in[0.5 \mathrm{~cm}$, $20 \mathrm{~cm}], L_{2} \in[5 \mathrm{~cm}, 30 \mathrm{~cm}], D_{c} \in[0.16 \mathrm{~cm}, 0.2 \mathrm{~cm}]$. The function of distance way by $X$ direction with changing $L_{1}, L_{2}$ and fixed $D_{c}=0.16 \mathrm{~cm}$ is presented in the left of Fig. 3 . The function of distance way by $X$ direction changing $L, D_{c}$ and fixed $L_{1}=2.5 \mathrm{~cm}$ is 
presented in the right of Fig. 3. The maximum area of the distance way by $X_{0}$ direction is shown by the red color area. The velocity $U$ and way distance processes by $X_{0}$ direction with or without optimal values of couples $\left(L_{1}, L_{2}, D_{c}\right)$ are shown in the Fig. 4 . By this figure it is easy to see that with the optimal values $L_{1}^{*}=0.5 \mathrm{~cm}, L_{2}^{*}=22.5 \mathrm{~cm}, D_{c}^{*}=0.16$ $\mathrm{cm}$ the way distance is maximum and reaches to $27.2 \mathrm{~m}$. With the two other parameters $\left(L_{1}, L_{2}, D_{c}\right)$ the way distances are less than $20 \mathrm{~m}$ but bigger than $17 \mathrm{~m}$.
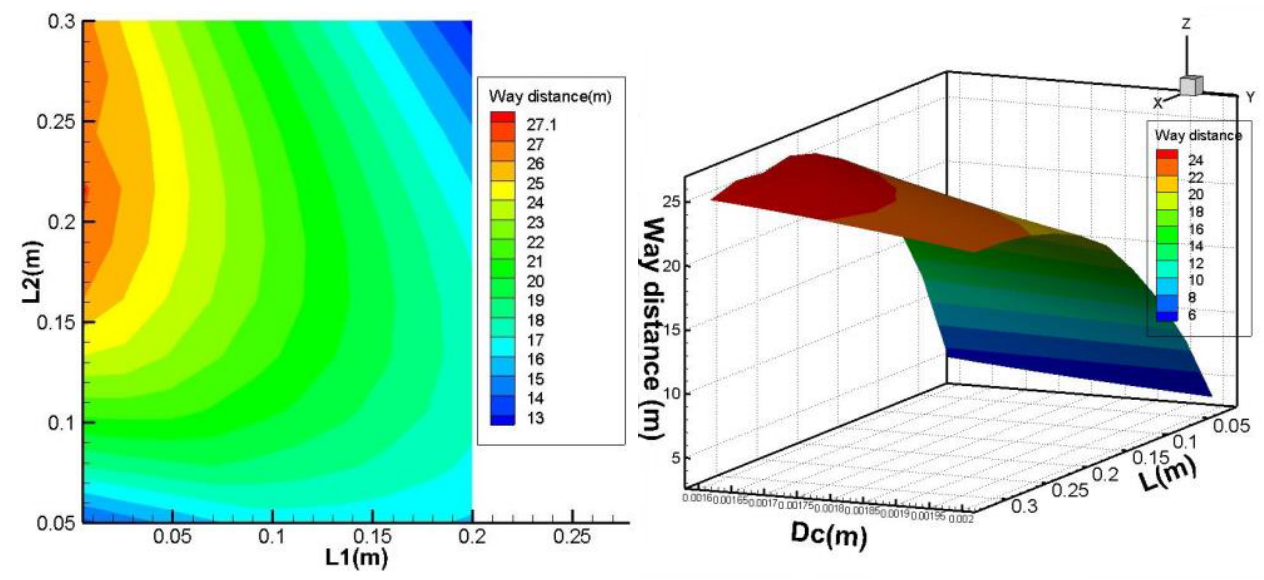

Fig. 3. The area of way distance by $X$ direction depending on $L_{1}$ and $L_{2}$ in optimal process with fixed $D_{c}=0.16 \mathrm{~cm}$ (left); The area of way distance by $X$ direction depending on $L$ and $D_{c}$ in optimal process with fixed $L_{1}=2.5 \mathrm{~cm}$ (right)
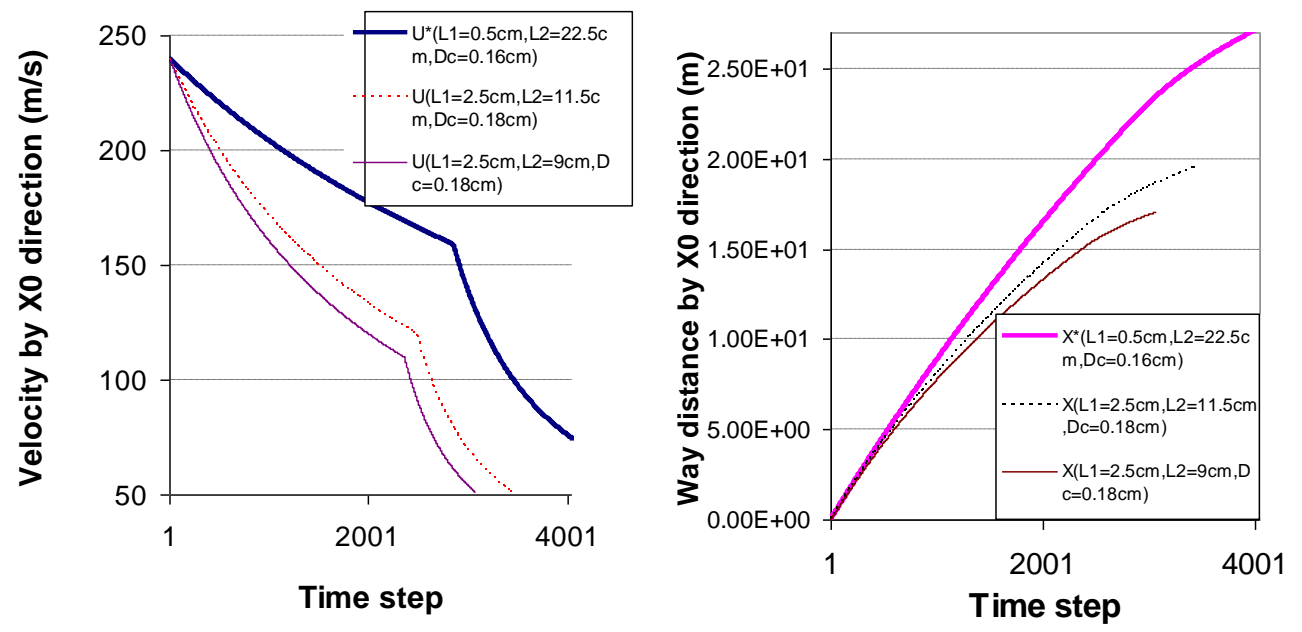

Fig. 4. Velocity by $X$ direction with or without optimal $\left(L_{1}, L_{2}, D_{c}\right)$ (left); Way distance by $X_{0}$ direction with or without optimal $\left(L_{1}, L_{2}, D_{c}\right)$ (right) 


\section{CONCLUSION}

In the paper the problem of determination of optimal geometric parameters of a slender body moving fast through water is considered by using the differential variation optimal method. The following observations are obtained:

- Fig. 3 shows that in the case $D_{c}=0.16 \mathrm{~cm}$ and $L_{1} \in[0.5 \mathrm{~cm}, 2.5 \mathrm{~cm}], L_{2} \in$ $[15 \mathrm{~cm}, 30 \mathrm{~cm}]$ the way distance is longer than $24 \mathrm{~m}$.

- Fig. 4 shows that by data assimilation method the chosen values $\left(L_{1}^{*}, L_{2}^{*}, D_{c}^{*}\right)$ make the way distance to be maximal. Thus, the optimal differential variation method can be used as a good tool to choose best geometric parameters in the problem of body running fast through water.

\section{ACKNOWLEDGEMENTS}

The authors acknowledge the support by the VAST.HDN.01/15-16 project.

\section{REFERENCES}

[1] R. Rand, R. Pratap, D. Ramani, J. Cipolla, and I. Kirschner. Impact dynamics of a supercavitating underwater projectile. In Proceedings of ASME Design Engineering Technical Conferences, Sacramento, CA, (1997), pp. 14-17.

[2] P. R. Garabedian. Calculation of axially symmetric cavities and jets. Pacific Journal of Mathematics, 6, (4), (1956), pp. 611-684. doi:10.2140/pjm.1956.6.611.

[3] T. Kiceniuk. An experimental study of the hydrodynamic forces acting on a family of cavityproducing conical bodies of revolution inclined to the flow. Technical report, CIT Hydrodynamics Report, (1954).

[4] I. N. Kirschner, N. E. Fine, J. S. Uhlman, and D. C. Kring. Numerical modeling of supercavitationg flow. Technical report, Paper presented at the RTO A VT Lecture Series on "Supercavitating Flows", held at the von Kcrmdn Institute (VKI) in Brussels, Belgium, (2001).

[5] G. V. Logvinovich. Hydrodynamics of free-boundary flows. Kiev, (1969).

[6] A. May. Water entry and the cavity-running behavior of missiles. Technical report, Navsea Hydroballistics Advisory Committee Silver Spring Md, (1975).

[7] B. Milwitzky. Generalized theory for seaplane impact. Technical report, NACA-TR-1103, (1952).

[8] N. A. Son, T. T. Ha, and D. N. Hai. A super cavity model of slender body moving fast in water. In Proceedings of the 10th National Congress on Mechanics, (2014), pp. 415-420.

[9] J.-L. Lions. Contrôle optimal de systemes gouvernés par des équations aux dérivées partielles. Paris: Dunod, (1968).

[10] J. L. Lions. Contrôlabilité exacte perturbations et stabilisation de systèmes distribués. Paris: Masson, (1988).

[11] G. I. Marchuk, V. I. Agoshkov, and V. P. Shutyaev. Adjoint equations and perturbation algorithms in nonlinear problems. CRC Press, (1996).

[12] I. Y. Gejadze, F.-X. Le Dimet, and V. Shutyaev. On optimal solution error covariances in variational data assimilation problems. Journal of Computational Physics, 229, (6), (2010), pp. 2159 2178. doi:10.1016/j.jcp.2009.11.028.

[13] I. Y. Gejadze, G. J. M. Copeland, F.-X. Le Dimet, and V. Shutyaev. Computation of the analysis error covariance in variational data assimilation problems with nonlinear dynamics. Journal of Computational Physics, 230, (22), (2011), pp. 7923-7943. doi:10.1016/j.jcp.2011.03.039. 
[14] R. Glowinski and J. L. Lions. Exact and approximate controllability for distributed parameter systems. Acta Numerica, 3, (1994), pp. 269-378. doi:10.1017/s0962492900002452.

[15] F.-X. L. Dimet, P. Ngnepieba, and V. Shutyaev. On error analysis in data assimilation problems. Russian Journal of Numerical Analysis and Mathematical Modelling, 17, (1), (2002), pp. 7198. doi:10.1515/rnam-2002-0105.

[16] F.-X. Le Dimet and V. Shutyaev. On deterministic error analysis in variational data assimilation. Nonlinear Processes in Geophysics, 12, (4), (2005), pp. 481-490. doi:10.5194/npg-12-4812005.

[17] F.-X. Le Dimet, V. P. Shutyaev, and T. H. Tran. General sensitivity analysis in data assimilation. Russian Journal of Numerical Analysis and Mathematical Modelling, 29, (2), (2014), pp. 107127. doi:10.1515/rnam-2014-0009.

[18] L. W. White, B. Vieux, D. Armand, and F. X. LeDimet. Estimation of optimal parameters for a surface hydrology model. Advances in Water Resources, 26, (3), (2003), pp. 337-348. doi:10.1016/s0309-1708(02)00189-6.

[19] M. Nahon. A simplified dynamics model for autonomous underwater vehicles. In Autonomous Underwater Vehicle Technology. IEEE, (1996), pp. 373-379, doi:10.1109/auv.1996.532437.

[20] S. S. Kulkarni and R. Pratap. Studies on the dynamics of a supercavitating projectile. Applied Mathematical Modelling, 24, (2), (2000), pp. 113-129. doi:10.1016/s0307-904x(99)00028-1. 